\title{
Reductions in Frontocortical Cytokine Levels are Associated with Long-Lasting Alterations in Reward Valuation after Methamphetamine
}

\author{
Alexandra Stolyarova', Andrew B Thompson', Ruth M Barrientos² and Alicia Izquierdo*,' \\ 'Department of Psychology, University of California, Los Angeles, Los Angeles, CA, USA; ${ }^{2}$ Department of Psychology and Neuroscience, University \\ of Colorado at Boulder, Boulder, CO, USA
}

\begin{abstract}
Alterations in reward valuation are thought to have a central role at all stages of the addiction process. We previously reported work aversion in an effortful T-maze task following a binge exposure to methamphetamine, and no such changes in effort following escalating doses. Limitations of the T-maze task include its two available options, with an effort requirement, in the form of increasing barrier height, varying incrementally as a function of time, and reward magnitudes held constant. Reward preferences and choices, however, are likely affected by the number of options available and the manner in which alternatives are presented. In the present experiment, we investigated the long-lasting, off-drug effects of methamphetamine on reward choices in a novel effortful maze task with three possible courses of action, each associated with different effort requirements and reward magnitudes. Neuroinflammatory responses associated with drug exposure, proposed as one of the mechanisms contributing to suboptimal choices on effort-based tasks, were also examined. We investigated region-specific changes in pro- and anti-inflammatory markers in the mesocorticolimbic pathway after methamphetamine, and their relationship with animals' reward choices. We observed long-lasting, increased sensitivity to differences in reward magnitude in the methamphetamine group: animals were more likely to overcome greater effort costs to obtain larger rewards on our novel effortful maze task. These behavioral changes were strongly predicted by pronounced decreases in frontocortical cytokines, but not amygdalar or striatal markers. The present results provide the first evidence that neuroinflammatory processes are associated with alterations in reward valuation during protracted drug withdrawal.

Neuropsychopharmacology (2015) 40, 1234-1242; doi:I0.1038/npp.2014.309; published online 17 December 2014
\end{abstract}

\section{INTRODUCTION}

Reward valuation is thought to have a central role at all stages of the addiction process: from initiation to compulsive use, abstinence and subsequent relapse, across species and substance classes (Hyman et al, 2006; Roesch et al, 2007; Diekhof et al, 2008). Drug addiction can be conceptualized as a maladaptive pattern of choices characterized by excessively high valuation of a reinforcer, combined with limited sensitivity to an increased cost in obtaining it (Bickel et al, 2011). Three types of cost are usually considered when analyzing choice behavior in animals: cost of time, physical or cognitive effort, and risk. Suboptimal choices on delay- (Bickel and Marsch, 2001; Mackillop et al, 2011) and risk-based (Kreek et al, 2005; Adlaf and Smart, 1983) decision-making tasks associated with drug exposure have been frequently reported.

* Correspondence: Professor A Izquierdo, Department of Psychology, University of California, Los Angeles, 1285 Franz Hall Box 951563, Los Angeles, CA 90095-1563, USA, Tel: + 310825 3459, Fax: + 310206 5895, E-mail: aizquie@psych.ucla.edu

Received 8 September 20।4; revised I I November 20।4; accepted 13 November 2014; accepted article preview online 20 November 2014
Effort discounting has, by comparison, received less attention in animal drug addiction research. We previously reported work aversion in an effortful T-maze task following a binge dose of methamphetamine (Kosheleff et al, 2011), but found no such changes in reward choices made in the face of increasing effort after subchronic escalating methamphetamine treatment (unpublished observation). Limitations of the T-maze task include its two available options, with an effort requirement, in the form of increasing barrier height, varying incrementally as a function of time, and reward magnitudes held constant. However, individual preferences and choice behavior are affected by the number of options available and the manner in which alternatives are presented (the 'framing effect'; Horowitz et al, 2007; Reutskaja and Hogarth, 2009; De Martino et al, 2006). To control for differences in processing cost changes, learning, and framing effects, we developed a novel effortful maze task with three possible courses of action, each associated with different effort requirements and reward magnitudes. For the present experiment, we chose a dosing regimen that produced an impairment on reversal learning even in the absence of appreciable changes in dopamine and serotonin transporter binding (Kosheleff et al, 2012).

Central immune signaling has been proposed as a mechanism of interest contributing to behavioral alterations 
following drug exposure (Clark et al, 2013; Sekine et al, 2008; Cadet and Bisagno, 2014; Coller and Hutchinson, 2012; Hutchinson and Watkins, 2014). Glial function has been linked to the development of rewarding properties of the drug and may have a role in the genetic predisposition to addiction (Beitner-Johnson et al, 1993; Narita et al, 2006). Peripheral inflammation has also been shown to affect effort-based decision making (Vichaya et al, 2014; Larson et al, 2002). However, little is known about neuroinflammation after repeated methamphetamine, and its effect on reward valuation in instrumental behavior. Thus, in the present investigation, we examined region-specific changes in pro- and anti-inflammatory markers in the mesocorticolimbic pathway (frontal cortex, amygdala, and striatum) after methamphetamine, and studied their relationship to animals' reward choices.

\section{MATERIALS AND METHODS}

\section{General}

Subjects were 12 ( saline $=6$, methamphetamine $=6$ ) adult male Long Evans rats (Charles River Laboratories), individually housed. Vivaria were maintained at $22^{\circ} \mathrm{C}$ under a 12/12-h light/dark cycle with lights on from 0600 to 1800 hours. All behavioral testing took place 5-6 days per week between 0800 and 1600 hours during the rats' inactive period, consistent with previous and ongoing studies in our lab. Rats were left undisturbed for 3 days after arrival to our facility to acclimate to the vivarium. Each rat was then handled for a minimum of 10 min once per day for 5 days. All rats were food restricted to no less than $85 \%$ of their free-feeding body weight to ensure motivation to work for food for a week prior and during the behavioral testing, whereas water was available ad libitum. On the last 2 days of food restriction prior to behavioral training, rats were fed twenty $1 / 2$ froot loops in their home cage to accustom them to food rewards. Weights were monitored daily to ensure a healthy body weight. Research protocols were approved by the Chancellor's Animal Research Committee at the University of California, Los Angeles.

\section{Drug Treatment}

Rats were given injections of methamphetamine $(n=6$, Sigma, St. Louis, MO; free base $/ \mathrm{kg}$, s.c.) starting at $0.3 \mathrm{mg} / \mathrm{kg}$ and escalating in $0.3-\mathrm{mg} / \mathrm{kg}$ increments per day, culminating in $6 \mathrm{mg} / \mathrm{kg}$, or physiological saline solution $(n=6,1 \mathrm{ml} /$ $\mathrm{kg}$, s.c.) 5 days per week for 4 weeks at 1100 hours. This 4 week treatment regimen was chosen for its similarity to other escalating methamphetamine and amphetamine protocols (Featherstone et al, 2008; Fletcher et al, 2007). Food intake was measured every week on withdrawal days. Behavioral testing began 5-7 days after the last injection.

\section{Behavioral Testing}

Testing apparatus. In the present experiment, we employed a novel task that utilized a maze with three possible courses of action, each associated with different effort requirements and reward magnitudes. Behavioral training and testing were conducted in a standard eight-arm radial maze with arms extending from a central arena with a diameter of $25 \mathrm{~cm}$. Arms were $50 \mathrm{~cm}$ long and $12 \mathrm{~cm}$ wide. The positions of extramaze cues remained constant throughout all phases of the experiment. The four arms nearest to the start arm were permanently blocked, leaving a start arm and three choice arms accessible to animals (Figure 1). One arm of the maze was randomly designated as a low-effort/reward (LER) arm, another as a mediumeffort/reward (MER) arm, and the third as a high-effort/ reward (HER) arm. The arm assignment was counterbalanced across animals, and held constant between sessions. The arm containing the low reward (LR) was unimpeded by a barrier, but to obtain the medium (MR) or high reward (HR), rats were required to climb a 20 - or $30-$ $\mathrm{cm}$ barrier, respectively. 'Froot loops' were given as food rewards during testing: a HR consisted of four $1 / 2$ froot loops (ie, two froot loops), a MR consisted of two $1 / 2$ froot loops (one froot loop), and a LR consisted of one $1 / 2$ froot loop. Between trials, the rat was removed from the maze and placed in Plexiglas holding chamber while the maze was wiped with $70 \%$ ethanol to eliminate residual odor cues.

Habituation. During the acclimation phase, five $1 / 2$ froot loops were placed into each arm of the maze (20 total). Each rat was individually placed into the maze and allowed to explore and eat froot loops freely. Criterion for advancement to the next phase was consumption of twenty $1 / 2$ froot loops within $15 \mathrm{~min}$.

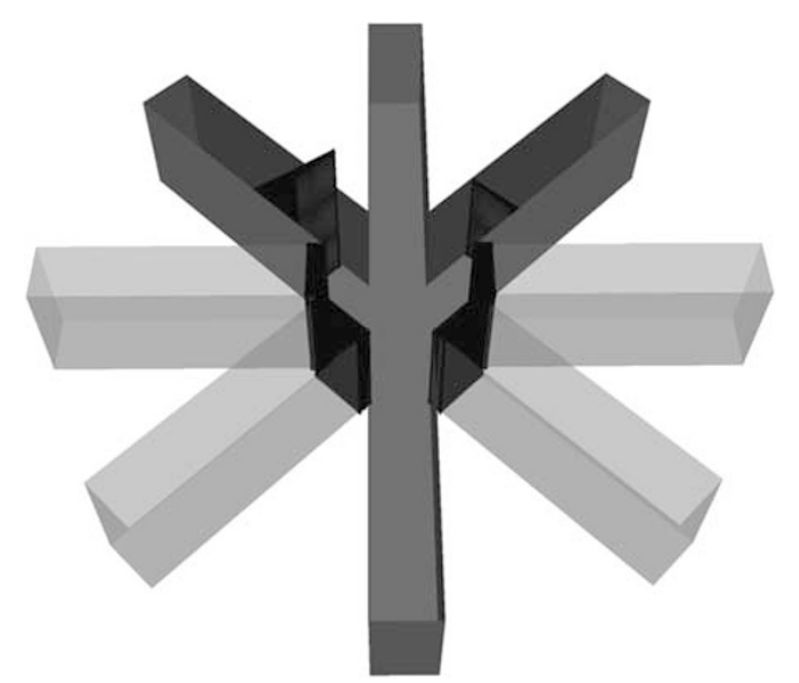

Figure I Novel effortful maze task. Behavioral training and testing were conducted in a standard eight-arm radial maze, with arms extending from a central arena with a diameter of $25 \mathrm{~cm}$. Arms were $50 \mathrm{~cm}$ long and $12 \mathrm{~cm}$ wide. The four arms nearest to the start arm were permanently blocked, leaving a start arm and three choice arms accessible to animals. One arm of the maze was randomly designated as a low-effort/reward (LER) arm, another as a medium-effort/reward (MER) arm, and the third as a higheffort/reward (HER) arm. The arm assignment was counterbalanced across animals, and held constant between sessions. The arm containing low reward was unimpeded by a barrier, but to obtain a medium or high reward, rats were required to climb a 20 - or 30-cm barrier, respectively. 'Froot loops' were given as food rewards during testing: a 'high reward' consisted of four $1 / 2$ froot loops (ie, two froot loops), a 'medium reward' consisted of two $1 / 2$ froot loops (one froot loop), and a 'low reward' consisted of one $1 / 2$ froot loop. 
Reward magnitude training: phase 1 . In this phase, one goal arm was baited with four $1 / 2$ froot loops (HR arm), another with two $1 / 2$ froot loops (MR arm), and the third arm with one $1 / 2$ froot loop (LR arm). Rats were allowed to sample freely from all arms for 10 trials. No barriers were present at this phase. Each trial lasted until the rat finished all the froot loops. Trials were separated by a 30-s inter-trial interval (ITI). The order of arm visits was recorded. Criterion for advancement to the next phase was completion of 10 trials within $30 \mathrm{~min}$.

Reward magnitude training: phase 2. This phase was similar to phase 1 of reward magnitude training, except that animals were allowed to visit only one arm per trial. Rats were removed from the maze as soon as the arm was chosen and the reward was consumed. Animals were given 10 trials per day separated by a 30 -s ITI. This phase marked the beginning of learning to visit only one arm, as well as continuing to learn each arm's associated reward values. Criterion for advancement to the next phase was choice of the HR arm on $80 \%$ or more of the trials for two consecutive days.

Alternating free-/forced-choice trials with barriers. During this phase, rats were required to climb barriers to achieve higher rewards. Each day of testing consisted of 10 free- and three forced-choice (one for each arm) trials, administered at the beginning. On forced-choice trials all goal arms except one were blocked. The order of arm presentation during forced-choice trials was counterbalanced between days. Upon eating the food reward, the rat was placed in a holding chamber for a 30-s ITI, during which the maze was wiped clean with $70 \%$ ethanol to prevent the rat's use of scent-guided choice. Rats were tested daily until stable baseline choice performance was established (choice preferences on free-choice trials did not significantly differ across three consecutive days).

Post-treatment testing. Seven days after the last injection, rats were tested again on the alternating free-/forced-choice trials with barriers to assess the effect of drug treatment on their choice preferences. After the post-treatment stable performance was established, a control task was administered in which work was equalized between the arms (identical to Reward magnitude training: phase 2).

\section{Tissue Dissection and Detection of Cytokines}

Rats were euthanized 1 day after the last day of behavioral testing (17 days after the final methamphetamine or saline treatment) with an overdose of sodium pentobarbital $(250 \mathrm{mg} / \mathrm{kg}$, i.p.) and decapitated. The brains were immediately extracted, flash frozen by immersion in isopentane over dry ice, and stored at $-80^{\circ} \mathrm{C}$ prior to dissections. The brains were then thawed in chilled artificial cerebrospinal fluid, and 2-mm-thick coronal sections of frontal cortex, striatum, and amygdala were further rapidly dissected, using a brain matrix, in saline with protease and phosphatase inhibitors (Halt, Thermo Scientific, Waltham, MA) at $4{ }^{\circ} \mathrm{C}$. Frontocortical dissections included ventral (orbital) and medial sectors of the frontal cortex, but excluded most lateral, posterior (agranular insular) regions.
Striatal dissections included both dorsal and ventral subregions. To prepare the tissues for the assays, $0.3 \mathrm{ml}$ (frontal cortex, striatum) or $0.2 \mathrm{ml}$ (amygdala) of a sonication buffer containing $50 \mathrm{mM}$ Tris base and a cocktail enzyme inhibitor ( $100 \mathrm{mM}$ amino- $n$-caproic acid, $10 \mathrm{mM}$ EDTA, $5 \mathrm{mM}$ benzamidine $\mathrm{HCl}$, and $0.2 \mathrm{mM}$ phenylmethyl sulfonyl fluoride) was added to each sample. Each tissue was mechanically sonicated for $20 \mathrm{~s}$ using an ultrasonic cell disrupter (Fisher Scientific, Pittsburgh, PA), centrifuged at $10000 \mathrm{~g}$ at $4{ }^{\circ} \mathrm{C}$ for $10 \mathrm{~min}$, and supernatants removed and stored at $4{ }^{\circ} \mathrm{C}$ until ELISA was performed. Bradford protein assays were also performed to determine total protein concentrations in each sample. IL-1 $\beta$, IL-6, IL-10 and TNF $\alpha$ cytokine protein levels were determined using a commercially available multiplex ELISA kit (Rat 4-plex Cat\# 114-944-1-AB; Aushon Biosystems, Billerica, MA). The assays were performed according to the manufacturer's instructions. All samples were run at a threefold dilution. The sensitivity of the multiplex assay is $6.25 \mathrm{pg} / \mathrm{ml}$ for IL-1 $\beta, 12.5 \mathrm{pg} / \mathrm{ml}$ for IL-6, $3.125 \mathrm{pg} / \mathrm{ml}$ for IL-10, and $25 \mathrm{pg} / \mathrm{ml}$ for TNF $\alpha$. The concentration of each cytokine is presented as $\mathrm{pg} / 100 \mu \mathrm{g}$ of total protein.

\section{Data Analyses}

Software package SPSS (SAS Institute, Version 16.0) was used for statistical analyses. The maze-choice data were first analyzed using multivariate ANOVA (MANOVA), with time (pre- and post treatment) and barrier height (LER, MER, HER) as within-subject and treatment group (methamphetamine $v s$ saline) as between-subject factors to probe for differences in choice preferences. Changes in choice preferences for each goal arm were subsequently analyzed using repeated-measures ANOVA (rmANOVA), with time (pre- and post treatment) as within-subject and treatment group as between-subject factors. Arm/reward choice preferences were assessed with rmANOVA analyses, with testing session as an additional within-subject factor. When significant interactions were found, post hoc simple effects were reported. Cytokine data for each brain region were analyzed using independent samples $t$-tests. Pearson correlation coefficients were calculated, and multiple linear regression analyses with the treatment group and IL- $1 \beta$, IL-6, IL-10 and TNF $\alpha$ levels as predictor variables were performed to examine the relationship between the cytokine levels and behavioral data. Statistical significance was noted when $p$-values $<0.05$ were observed. For both pre- and post-treatment testing, data from 11 animals were analyzed owing to loss of one animal in the methamphetamine group.

\section{RESULTS}

\section{Effortful maze Performance}

A significant effect of treatment group on arm choices was observed $(\mathrm{F}(5,5)=34.366, p<0.01$, Wilk's $\Lambda=0.03$, partial $\eta^{2}=0.972$ ). Changes in choice preferences for each goal arm were subsequently analyzed (results provided below). There were no pre-existing group differences and no preference for any of the goal arms prior to treatment: all animals distributed their choices uniformly across the available reward options (Figure 2a). However, there was a strong post-treatment preference for the MER arm compared with 
a
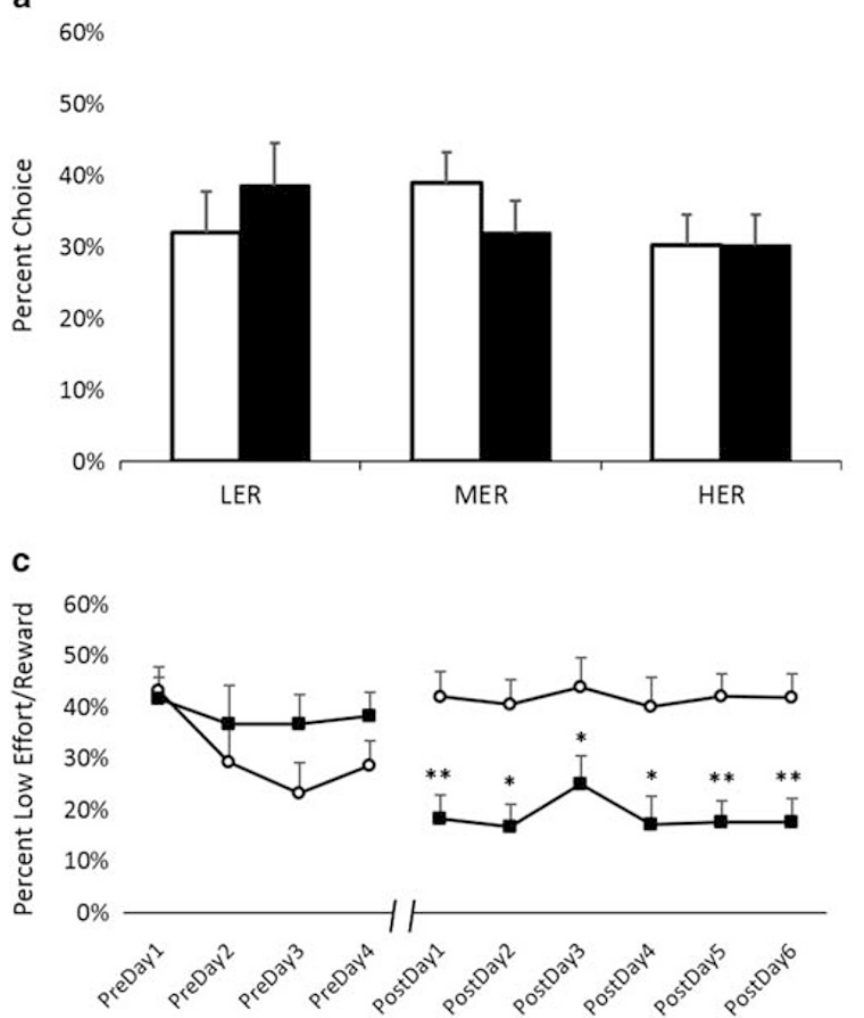

b
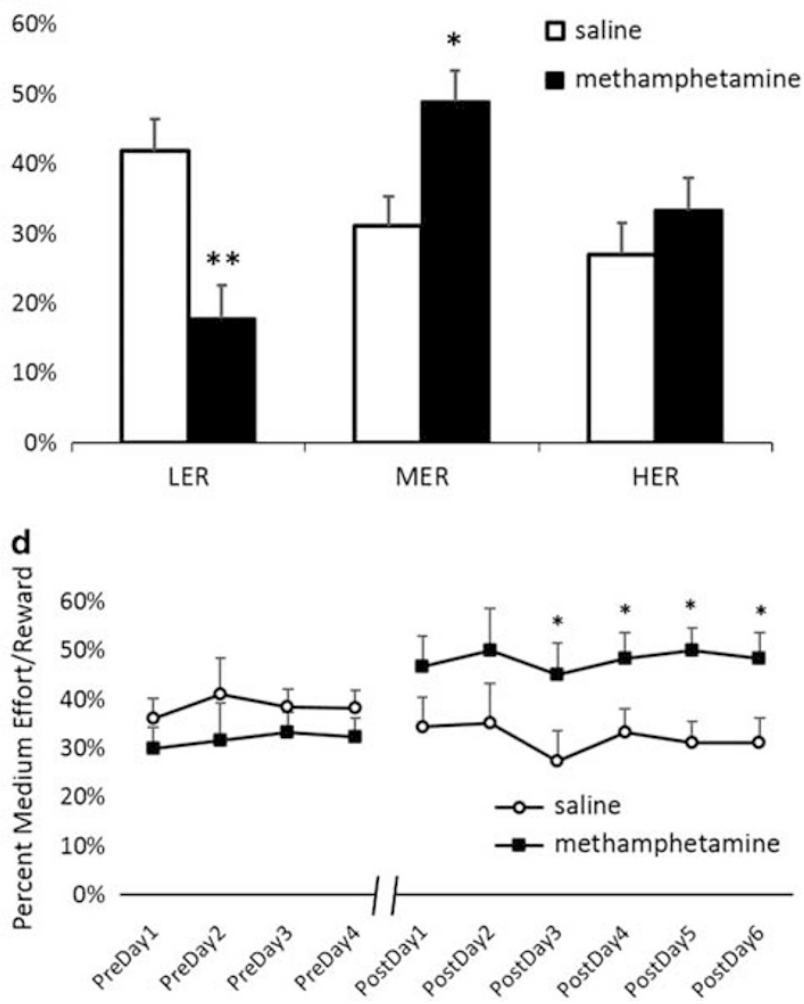

Figure 2 Methamphetamine pretreatment led to an increase in medium-effort/reward (MER) choices in preference to low-effort/reward (LER) option, leaving high-effort/reward (HER) choices unaffected. Bar graphs represent percent HER, medium effort/reward (MER), and LER pre- (a) and post-treatment (b) + SEM. (a) There were no pre-existing group differences and no preference for any of the goal arms prior to treatment; all animals distributed their choices uniformly across the reward arms. (b) There was a strong post-treatment preference for the MER arm compared with the LER arm ( $p<0.0 \mathrm{I}$ ) in methamphetamine animals, which was not present in the saline-treated group. Methamphetamine-treated animals chose the MER option more frequently compared with the saline group ( $p<0.05$ ), and shifted away from the LER option $(p<0.0 I)$. Groups were not different on HER choices. Methamphetaminepretreated animals shift away from LER immediately, whereas MER preference increases as a result of repeated training. (c) The shift-away from LER was present from the beginning of the post-treatment testing (starting day I, $p<0.0 \mathrm{I}$ ). (d) The MER choice increases became significant on day 3 ( $p<0.05$ ). * $p<0.05, * * * 0.01$.

the LER arm $(p<0.01)$ in methamphetamine animals, which was not present in the saline-treated group (Figure $2 b$ ). Additional trial-by-trial analyses of choice behavior revealed no group differences in patterns of arm choices. Animals did not exhibit any first-entry arm preference. There was also no effect of forced choice on subsequent choices, and the animals were not more likely to repeat the last forced choice than they were to choose a different arm. All animals exhibited a greater preference for higherreward/higher-effort options at the beginning of the session and decreased their willingness to exert greater effort for larger rewards with session progression, as evidenced by the greater probability of animals switching to less-effortful options on subsequent trials (main effect of choice $\mathrm{F}(1,9)=20.132, p<0.01)$. All animals were also more likely to alternate than repeat the arm choice on subsequent trials (main effect of choice $\mathrm{F}(1,9)=198.45, p<0.01$ ), but drug treatment did not affect a tendency for arm choice perseveration.

Methamphetamine pretreatment led to an increase in MER choices over the LER option, leaving HER choices unaffected. Methamphetamine-induced effects were ob- served on LER and MER, but not HER choices. For LER choices, there was no main effect of time or treatment group, but a significant time by treatment group interaction $(\mathrm{F}(1,5)=40.262, p<0.01)$ was observed. Post hoc analyses revealed a simple main effect of treatment group on posttreatment choices $(p<0.01)$, with methamphetamine-pretreated animals shifting away from the LER option (Figure 2b). For MER choices, analyses revealed no main effect of time or treatment group, but a significant time by treatment group interaction $(\mathrm{F}(1,5)=39.406, p<0.01)$. Post hoc comparisons showed that methamphetamine pretreated animals chose the MER option significantly more often following methamphetamine treatment $(p<0.05)$ (Figure 2b). No significant effects were observed on HER choices (Figure $2 \mathrm{a}$ and $\mathrm{b}$ ), as further evidence that the increased preference for the MER arm in methamphetamine-pretreated animals was a result of shifting away from the LER option.

Methamphetamine-pretreated animals shift away from LER immediately, whereas MER preference increases as a result of repeated training. For LER choices, rmANOVA revealed a significant main effect of the testing day 
$(\mathrm{F}(9,81)=2.276, p<0.05)$ and significant day by treatment group interaction $(\mathrm{F}(9,81)=7.818, p<0.01)$, but no main effect of treatment group. For MER choices, rmANOVA resulted in no main effect of the testing day or treatment group, but a significant day by treatment group interaction $(\mathrm{F}(9,81)=8.054, p<0.01))$. Post hoc analyses further showed that the shift away from LER was present from the beginning of the post-treatment testing (starting day 1 , $p<0.01$; Figure 2c), whereas the significant increase in MER choices first emerged on day 3 ( $p<0.05$; Figure $2 \mathrm{~d})$.

\section{General}

Main effect of barrier height on latencies to complete the trial was observed $(\mathrm{F}(2,32)=452.46, p<0.01)$; all animals took significantly longer to complete the HER compared with MER and LER, and MER compared with LER trials (all $p$-values $<0.01$ ). There was no effect of treatment on latencies to complete the trial for any type of reward choice. There were also no differences in the number of days that the animals required to reach stable performance. Performance on the control task was identical between groups. Methamphetamine treatment did not affect animals' weight or food intake (Figure 3).
Frontocortical Cytokine Levels are Decreased Following Methamphetamine Treatment and are Correlated with LER and MER Choices

Significant reductions in frontocortical IL- $1 \beta(t(9)=2.2341$, $p<0.05)$, IL-6 $(t(9)=2.308, p<0.05)$, IL-10 $(t(9)=2.432$, $p<0.05)$, and TNF $\alpha(t(9)=2.454, p<0.05)$ in the methamphetamine group were found (Figure $4 \mathrm{a}$ ). The increases in cytokine levels in amygdala were not significant (Figure 4b), and there were no between-group differences in striatal cytokine levels (Figure 4c). Levels of all of the cytokines within one brain region were strongly correlated with each other; this was true for both treatment groups considered independently and for all the brain regions examined (all $r$-values $>0.9$; all $p$-values $<0.01$ ), except IL-10 levels within striatum, which did not correlate with any other measurement within this brain region.

Multiple linear regression analyses with the treatment group and frontocortical IL-1 $\beta$, IL-6, IL-10, and TNF $\alpha$ levels as predictor variables further revealed that frontocortical cytokine levels significantly predicted post-treatment MER $\left(\mathrm{F}(5,10)=4.857, p<0.05, R^{2}=0.822\right)$ choices. The levels of each of the frontocortical cytokines examined, IL-1 $\beta$ (Figure 5a and b), IL-6, IL-10, and TNF $\alpha$, were indepen-
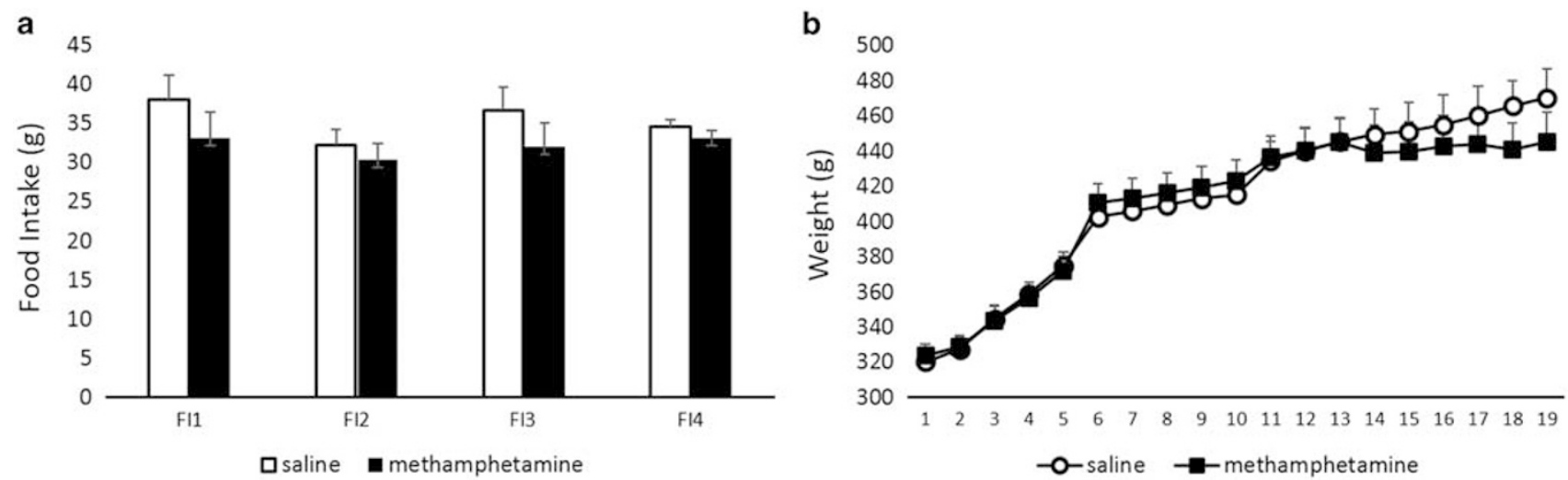

Figure 3 Methamphetamine treatment did not affect animals' food intake or body weight. Graphs represent amount of food (g) consumed in I day during acute withdrawal (a) or daily body weight (b) during the treatment period + SEM. No effect of the treatment group on animals' weight or food intake was observed; the differences in reward choices were not due to differential effects on motivation resulting from food restriction.
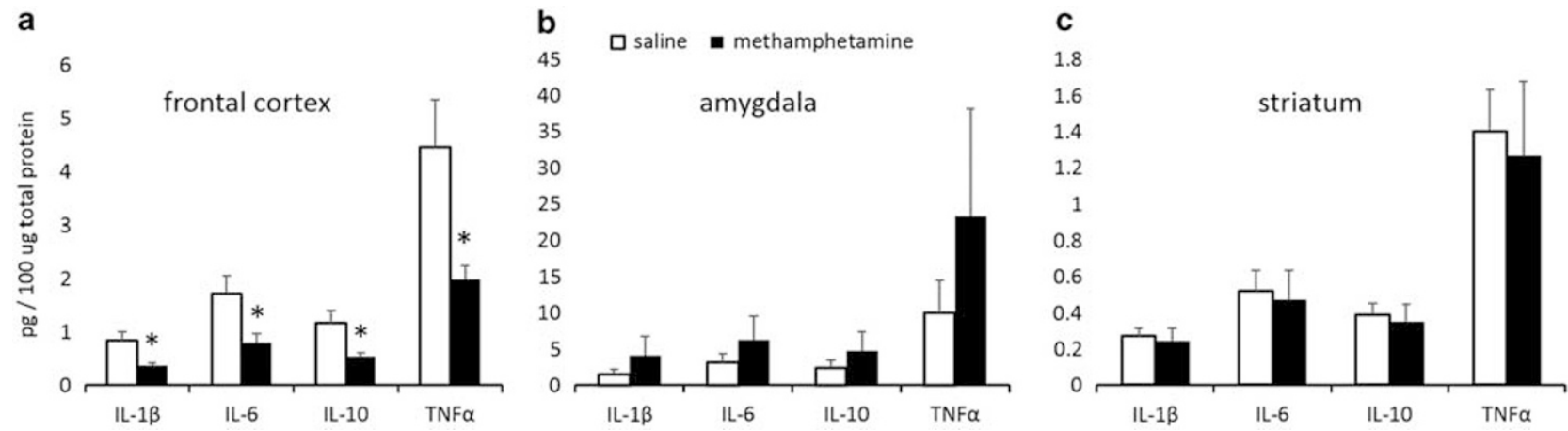

Figure 4 Frontocortical cytokine levels are decreased following methamphetamine treatment. Bar graphs represent concentration levels of each cytokine in $100 \mu \mathrm{g}$ of total protein + SEM. (a) Significant reduction in frontocortical interleukin (IL)-I $\beta(t(9)=2.234 \mid, p<0.05), I L-6(t(9)=2.308, p<0.05)$, IL-I0 $(t(9)=2.432, p<0.05)$, and tumor necrosis factor alpha (TNF $\alpha)(t(9)=2.454, p<0.05)$ in the methamphetamine group was found. (b) The increases in cytokine levels in amygdala were not significant, and (c) there were no between-group differences in striatal cytokine levels. $* p<0.05$. 

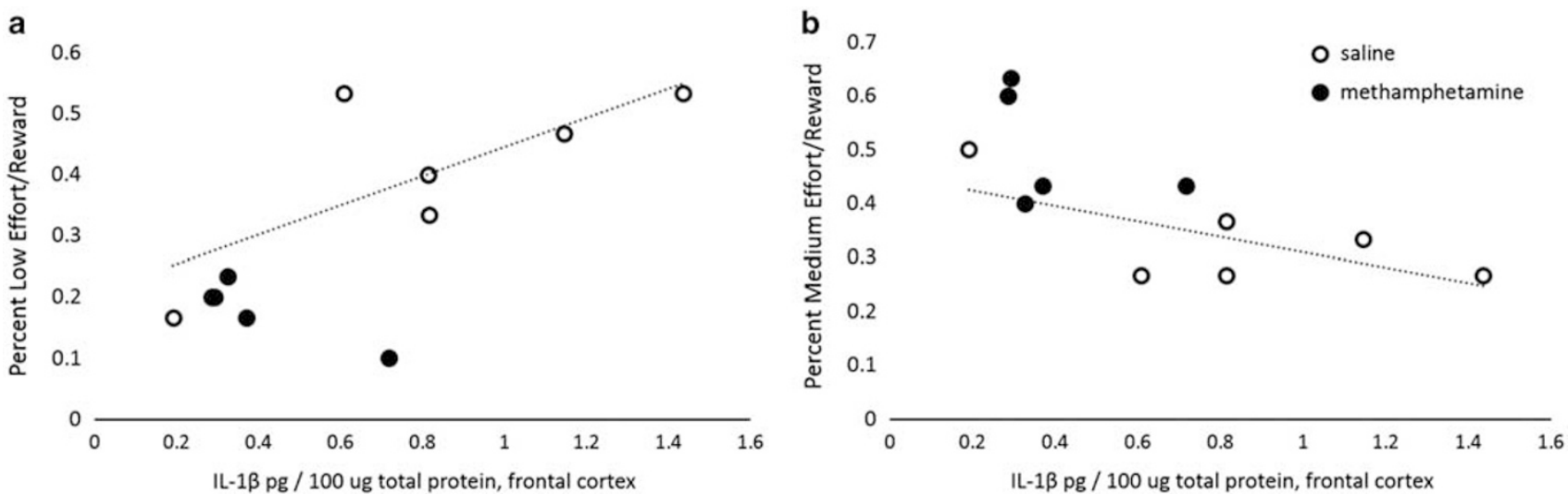

Figure 5 The alterations in reward valuation were associated with reductions in frontocortical cytokine levels. The figure illustrates significant strong positive correlations between frontocortical interleukin (IL)- I $\beta$ and (a) low-effort/reward (LER) choices $(r(I)=0.782$, $p<0.0$ I), as well as significant strong negative correlations between frontocortical IL-I $\beta$ and (b) medium-effort/reward (MER) choices $(r(I I)=-0.73, p=0.0 \mathrm{II})$. There was a statistical trend for positive correlations between frontocortical IL-I $\beta$, IL-6, IL- I , and tumor necrosis factor alpha (TNF $\alpha$ ) levels and LER choices $(0.05<$ all $p$-values $<0.1$ ), and negative correlations between frontocortical cytokines and MER choices $(0.06<$ all $p$-values $<0.15)$ in the saline group considered independently. The regression lines are given for the saline group only, and the methamphetamine group values are shown for comparison.

dently associated with LER choices (all $r$-values $>0.7$; all $p$-values <0.01), and negatively correlated with MER choices (all $r$-values $<-0.7$; all $p$-values $<0.05$ ). There was a statistical trend for positive correlations between frontocortical IL-1 $\beta$, IL-6, IL-10, and TNF $\alpha$ levels and LER choices $(0.05<$ all $p$-values $<0.1)$, and negative correlations between frontocortical cytokines and MER choices $(0.06<$ all $p$-values $<0.15)$ in the saline group considered independently. Pronounced decreases in cytokine levels and minimal variation in the methamphetamine group precluded correlational analyses. Interestingly, frontocortical cytokine levels were also negatively correlated with the change in MER choices (ie, difference between pre- and post-treatment; all $r$-values $<-0.67$; all $p$-values $<0.05$ ), whereas levels of cytokines in amygdala were positively correlated with this change (all $r$-values $>0.66$; all $p$-values $<0.05)$. The negative correlations between frontocortical cytokines IL-10 and TNF $\alpha$ were significant in the saline group considered separately (all $p$-values $<0.05$; trend for IL-1 $\beta: p=0.08$; IL-6: $p=0.05$ ), and there was a trend for positive correlations between cytokine levels in amygdala and the change in MER choices in the saline group $(0.05<$ all $p$-values $<0.1)$. No correlations between food intake or body weight and cytokine levels within any of the brain regions were observed.

\section{DISCUSSION}

Work aversion in a T-maze effort-discounting task at barrier heights of 25 and $30 \mathrm{~cm}$ (Kosheleff et al, 2011) was demonstrated after a binge regimen of methamphetamine. However, previous findings by Floresco and Whelan (2009) demonstrating comparable rates of effort discounting after repeated amphetamine treatment, and our laboratory showing no differences in choice preferences on a T-maze task after escalating methamphetamine (unpublished observation), indicate that effort-cost processing is not affected by repeated (meth)amphetamine exposure. In contrast, the results of the present investigation demonstrate an increased sensitivity to differences in reward magnitude in the methamphetamine group, making animals more likely to overcome greater effort costs to obtain larger rewards in a novel effortful maze task. In addition, we provide the first evidence for enduring region-specific neuroimmune changes as a consequence to escalating methamphetamine, which are associated with goal-directed, reward choice alterations in the long term.

\section{Methamphetamine Pretreatment Produced Long- Lasting Alterations in Reward Valuation}

Individual preferences and choice behavior are affected by the number of alternative options available and the manner in which alternatives are presented (Horowitz et al, 2007; Reutskaja and Hogarth, 2009; De Martino et al, 2006), as well as by learning and experience. To control for differences in reward learning and to better assess valueguided decision making, we trained animals on a novel effortful task with three possible courses of action, each associated with different effort requirements and reward magnitudes, until stable choice performance was established. This task, with increased option space, better mimics decision-making problems faced by animals in their natural environment. There were no pre-existing preferences for any of the goal arms: all animals distributed their choices uniformly across the available reward options. In addition, no group differences in the pattern or order of choices were observed: all animals showed greater preference for highereffort/higher-reward options at the beginning of a session. Increased preference for MER and decreased choice of the LER option appeared during post-treatment testing in the methamphetamine, but not the saline, treatment group. Methamphetamine-pretreated animals were more likely to overcome greater effort costs to obtain larger rewards compared with the saline group, consistent with previous work by our laboratory employing an identical treatment regimen (Stolyarova et al, 2014) and others (Dezfouli et al, 
2009) showing increased reward sensitivity in drugpretreated animals. Notably, these behavioral alterations were observed outside of the acute withdrawal period, suggesting that methamphetamine exposure has longlasting effects on reward choice preferences. The observed methamphetamine-induced shifts in choice behavior were not associated with changes in primary food motivation.

Interestingly, HER choices were unaffected by methamphetamine treatment. The choices in the task employed in the present experiment are based on the comparison of the relative differences in both reward magnitude and physical demands. The magnitude of the reinforcement associated with two $1 / 2$ froot loops in food-restricted animals might be perceived as sufficiently high, and further increases in reward size may be discounted more steeply by increases in effort cost. In addition, the effort- and time-cost sensitivity, which has been shown to be affected by methamphetamine exposure (Monterosso et al, 2007; Hoffman et al, 2006), are confounded in the present task, as increases in barrier height introduce a delay to obtain the reward. Finally, climbing the high barrier, which extends $10 \mathrm{~cm}$ above the maze walls, may be more anxiogenic to methamphetaminepretreated animals. Indeed, methamphetamine-induced acute and long-term increases in anxiety levels have been reported (London et al, 2004; Kitanaka et al, 2008; Darke et al, 2008; Pometlová et al, 2012; Akindipe et al, 2014). Stress and anxiety, in turn, have been implicated in alterations in cost-benefit decision making, with acute stress exposure leading to decreases in the preference for the more costly reward (Shafiei et al, 2012). Although not addressed in the present investigation, all the aforementioned factors may impose an additional demand and increase the cost associated with climbing the high compared with medium barrier, masking increases in motivation to obtain the larger reward.

The observed patterns of behavioral changes could be due to termination of drug administration and removal of the drug reinforcer, rather than a direct consequence of drug exposure. Interactions between drug and non-drug (natural) reinforcers on the establishment and maintenance of operant behavior have been previously reported. Carroll et al (1989) showed that removal of the first reinforcer led to enhanced acquisition of operant behavior for the second reinforcer (either drug or natural reward). Taken together with previous results from our laboratory demonstrating increased sensitivity to positive reward feedback (Stolyarova et $a l, 2014)$, this adds to the evidence for increased reward sensitivity as a result of drug exposure/termination.

\section{The Alterations in Reward Valuation in the Methamphetamine Group were Associated with Reductions in Frontocortical Cytokine Levels}

The methamphetamine-induced alterations in reward valuation were accompanied by pronounced decreases is frontocortical cytokine levels. The role for central immune signaling in the modulation of drug-induced neuronal and behavioral responses has been recently suggested (Coller and Hutchinson, 2012; Hutchinson and Watkins, 2014). Glial function has been linked to genetic differences in drug response, and there is demonstrated involvement of astrocyte-specific soluble factors, induced by drug expo- sure, in the development of the rewarding effects of methamphetamine at different doses (Narita et al, 2005; Narita et al, 2006). Systemic elevation of pro-inflammatory cytokines has been shown to affect effort-based decision making, shifting the choice behavior toward LER options and reducing the tendency to work for reinforcers (Larson et al, 2002; Nunes et al, 2014; Vichaya et al, 2014), but the involvement of centrally acting cytokines in choice behavior on effortful tasks had not been demonstrated. Here we provide novel evidence implicating cytokines within the frontal cortex in alterations in value-based decision making after methamphetamine exposure.

Although acute methamphetamine administration has been shown to induce potent increases in inflammatory cytokines (Flora et al, 2002; Gonçalves et al, 2008), the results of the present investigation demonstrate pronounced decreases in cortical cytokines 17 days after subchronic escalating methamphetamine treatment in rats, as well as non-significant increases of cytokine levels in amygdala. These results are in accordance with a previous report by Loftis et al (2011) demonstrating decreased levels of IL- $1 \beta$ in frontal cortex of mice pre-treated with methamphetamine $(1 \mathrm{mg} / \mathrm{kg}$ for 7 days $) 72 \mathrm{~h}$ after the last injection. However, in the investigation by Loftis et al (2011), the differences in cytokine levels were no longer present 21 days after treatment. The persistence of neuroimmune changes in the present study may be attributed to a significantly longer duration of treatment, escalating regimen, or the highest administered dose. One possible explanation for the observed decreases is that chronic methamphetamine administration may robustly dysregulate IL-1 $\beta$ receptor expression, causing enduring reductions of the protein. However, this possibility and its time course remain to be elucidated in a future study. Cytokine expression patterns are known to exhibit variable time courses in response to various immune challenges, conditions, and disease states (Barrientos et al, 2009; Csontos et al, 2010; Thijs and Hack, 1995). Anti-inflammatory cytokines are released as part of the homeostatic response, and often exhibit a temporal profile that overlaps with that of pro-inflammatory cytokines (Henry et al, 2009; Kamm et al, 2006; Turrin et al, 2001). Thus, if samples are collected at one of those overlapping time points, pro- and anti-inflammatory cytokines can exhibit changes in parallel, as was observed here.

Notably, protein levels of all cytokines within one brain region were strongly correlated, providing evidence for global effects of methamphetamine on glial function. The lack of changes in cytokine levels within striatum and nonsignificant between-group differences in amygdala may be attributed to the duration of abstinence from the drug, as the neuroinflammatory responses may subside over longer periods of abstinence (Sekine et al, 2008). Future investigations could assess larger sample sizes, sex differences, and the time course of drug-induced neuroinflammatory responses.

Frontocortical cytokine levels were positively correlated with LER and negatively correlated with MER choices. In addition, MER choices were associated with pronounced decreases in frontocortical cytokines, providing evidence that neuroinflammatory processes within frontocortical regions may contribute to alterations in reward evaluation during withdrawal, when re-experience with the drug can 
lead to rapid reinstatement. Importantly, our frontocortical dissections included the ventromedial region, which has been implicated in the evaluation of goals in the presence of several good options and in regret associated with comparison of the selected and the forgone choice (Blair et al, 2006; Chau et al, 2014). Interestingly, frontocortical cytokine levels were also negatively correlated with the change in MER choices (ie, difference between pre- and post-treatment), whereas levels of cytokines in amygdala were positively correlated with this change. These results show that cytokines within both amygdala and frontal cortex contribute to pre- and post-treatment changes in subjective values of reward options, whereas betweenoption choices following repeated training are more influenced by frontocortical neuroimmune responses. This interpretation is consistent with the idea that amygdala is critical in updating reward values, but is no longer recruited when decisions depend on stable preferences or familiar outcomes (Izquierdo and Murray, 2007).

In summary, we provide the first evidence that neuroinflammatory processes, specifically reduction of frontocortical cytokine levels, may contribute to alterations in reward valuation during protracted methamphetamine withdrawal. The mechanism by which changes in central cytokines contribute to behavioral alterations are not well understood. Previous evidence suggests that interleukins may be required for normal neuronal function, and that the effect of regional changes in cytokine concentration follows an inverted U-shape curve, with any deviation (excess or reduction) from the physiological range producing an impairment (Goshen et al, 2007). Pro-inflammatory cytokines can have a direct effect on neuronal excitability by altering GABA-ergic and glutamatergic signaling (Stellwager et al, 2005). In addition, cytokines may interact with monoamine transmission, by affecting dopamine metabolism (Song et al, 2006; Miller et al, 2013). The effect on dopaminergic transmission is particularly intriguing given that in a study by Nunes et al (2014), the IL-1 $\beta$-induced changes in effortful choice behavior resembled those produced by dopamine antagonism or depletion.

\section{FUNDING AND DISCLOSURE}

This work was supported by UCLA's Division of Life Sciences Recruitment and Retention fund (Izquierdo). Partial support for ABT was provided by the TNDA T32 DA024635 (Thompson). The authors declare no conflict of interest.

\section{ACKNOWLEDGEMENTS}

We thank Adrianna De La Torre for help with collecting the food intake data.

\section{REFERENCES}

Adlaf EM, Smart RG (1983). Risk-taking and drug-use behavior: an examination. Drug Alcohol Depend 11: 287-296.

Akindipe T, Wilson D, Stein DJ (2014). Psychiatric disorders in individuals with methamphetamine dependence: prevalence and risk factors. Metab Brain Dis 29: 351-357.
Barrientos RM, Frank MG, Hein AM, Higgins EA, Watkins LR, Rudy JW et al (2009). Time course of hippocampal IL-1 beta and memory consolidation impairments in aging rats following peripheral infection. Brain Behav Immun 23: 46-54.

Beitner-Johnson D, Guitart X, Nestler EJ (1993). Glial fibrillary acidic protein and the mesolimbic dopamine system: regulation by chronic morphine and Lewis-Fischer strain differences in the rat ventral tegmental area. J Neurochem 61: 1766-1773.

Bickel WK, Marsch LA (2001). Toward a behavioral economic understanding of drug dependence: delay discounting processes. Addiction 96: 73-86.

Bickel WK, Jarmolowicz DP, Mueller ET, Gatchalian KM (2011). The behavioral economics and neuroeconomics of reinforcer pathologies: implications for etiology and treatment of addiction. Curr Psychiatry Rep 13: 406-415.

Blair K, Marsh AA, Morton J, Vythilingam M, Jones M, Mondillo K et al (2006). Choosing the lesser of two evils, the better of two goods: specifying the roles of ventromedial prefrontal cortex and dorsal anterior cingulate in object choice. J Neurosci 26: 11379-11386.

Cadet J, Bisagno V (2014). Glial-neuronal ensembles: partners in drug addiction-associated synaptic plasticity. Front Pharmacol 5: 204.

Carroll ME, Lac ST, Nygaard SL (1989). A concurrently available nondrug reinforcer prevents the acquisition or decreases the maintenance of cocaine-reinforced behavior. Psychopharmacology (Berl) 97: 23-29.

Chau BK, Kolling N, Hunt LT, Walton ME, Rushworth MF (2014). A neural mechanism underlying failure of optimal choice with multiple alternatives. Nat Neurosci 17: 463-470.

Clark KH, Wiley CA, Bradberry CW (2013). Psychostimulant abuse and neuroinflammation: emerging evidence of their interconnection. Neurotox Res 23: 174-188.

Coller JK, Hutchinson MR (2012). Implications of central immune signaling caused by drugs of abuse: mechanisms, mediators and new therapeutic approaches for prediction and treatment of drug dependence. Pharmacol Ther 134: 219-245.

Csontos C, Foldi V, Palinkas L, Bogar L, Roth E, Weber G et al (2010). Time course of pro- and anti-inflammatory cytokine levels in patients with burns-prognostic value of interleukin-10. Burns 36: 483-494.

Darke S, Kaye S, McKetin R, Duflou J (2008). Major physical and psychological harms of methamphetamine use. Drug Alcohol Rev 27: 253-262.

De Martino B, Kumaran D, Seymour B, Dolan RJ (2006). Frames, biases, and rational decision-making in the human brain. Science 313: 684-687.

Diekhof EK, Falkai P, Gruber O (2008). Functional neuroimaging of reward processing and decision-making: a review of aberrant motivational and affective processing in addiction and mood disorders. Brain Res Rev 59: 164-184.

Dezfouli A, Piray P, Keramati MM, Ekhtiari H, Lucas C, Mokri AA (2009). Neurocomputational model for cocaine addiction. Neural Comput 21: 2869-2893.

Featherstone RE, Rizos Z, Kapur S, Fletcher PJ (2008). A sensitizing regimen of amphetamine that disrupts attentional set-shifting does not disrupt working or long-term memory. Behav Brain Res 189: 170-179.

Fletcher PJ, Tenn CC, Sinyard J, Rizos Z, Kapur S (2007). A sensitizing regimen of amphetamine impairs visual attention in the 5-choice serial reaction time test: reversal by a D1 receptor agonist injected into the medial prefrontal cortex. Neuropsychopharmacology 32: 1122-1132.

Flora G, Lee YW, Nath A, Maragos W, Hennig B, Toborek M (2002). Methamphetamine-induced TNF-alpha gene expression and activation of AP-1 in discrete regions of mouse brain: potential role of reactive oxygen intermediates and lipid peroxidation. Neuromolecular Med 2: 71-85. 
Floresco SB, Whelan JM (2009). Perturbations in different forms of cost/benefit decision making induced by repeated amphetamine exposure. Psychopharmacology (Berl) 205: 189-201.

Gonçalves J, Martins T, Ferreira R, Milhazes N, Borges F, Ribeiro CF et al (2008). Methamphetamine-induced early increase of IL-6 and TNF-alpha mRNA expression in the mouse brain. Ann N Y Acad Sci 1139: 103-111.

Goshen I, Kreisel T, Ounallah-Saad H, Renbaum P, Zalzstein Y, Ben-Hur $\mathrm{T}$ et al (2007). A dual role for interleukin-1 in hippocampal-dependent memory processes. Psychoneuroendocrinology 32: 1106-1115.

Henry CJ, Huang Y, Wynne AM, Godbout JP (2009). Peripheral lipopolysaccharide (LPS) challenge promotes microglial hyperactivity in aged mice that is associated with exaggerated induction of both pro-inflammatory IL-1beta and anti-inflammatory IL-10 cytokines. Brain Behav Immun 23: 309-317.

Hoffman WF, Moore M, Templin R, McFarland B, Hitzemann RJ, Mitchell SH (2006). Neuropsychological function and delay discounting in methamphetamine-dependent individuals. Psychopharmacology (Berl) 188: 162-170.

Horowitz JK, List J, McConnell KE (2007). A test of diminishing marginal value. Economica 74: 650-663.

Hutchinson MR, Watkins LR (2014). Why is neuroimmunopharmacology crucial for the future of addiction research? Neuropharmacology 76: 218-227.

Hyman SE, Malenka RC, Nestler EJ (2006). Neural mechanisms of addiction: the role of reward-related learning and memory. Annu Rev Neurosci 29: 565-598.

Izquierdo A, Murray EA (2007). Selective bilateral amygdala lesions in rhesus monkeys fail to disrupt object reversal learning. J Neurosci 27: 1054-1062.

Kamm K, Vanderkolk W, Lawrence C, Jonker M, Davis AT (2006). The effect of traumatic brain injury upon the concentration and expression of interleukin-1beta and interleukin-10 in the rat. J Trauma 60: 152-157.

Kitanaka J, Kitanaka N, Takemura M (2008). Neurochemical consequences of dysphoric state during amphetamine withdrawal in animal models: a review. Neurochem Res 33: 204-219.

Kosheleff AR, Grimes M, O'Dell SJ, Marshall JF, Izquierdo A (2011). Work aversion and associated changes in dopamine and serotonin transporter after methamphetamine exposure in rats. Psychopharmacology (Berl) 219: 411-420.

Kosheleff AR, Rodriguez D, O'Dell SJ, Marshall JF, Izquierdo A (2012). Comparison of single-dose and escalating methamphetamine administration on reversal learning in rats. Psychopharmacology (Berl) 224: 459-467.

Kreek MJ, Nielsen DA, Butelman ER, LaForge KS (2005). Genetic influences on impulsivity, risk taking, stress responsivity and vulnerability to drug abuse and addiction. Nat Neurosci 8: $1450-1457$.

Larson SJ, Romanoff RL, Dunn AJ, Glowa JR (2002). Effects of interleukin-1beta on food-maintained behavior in the mouse. Brain Behav Immun 16: 398-410.

Loftis JM, Choi D, Hoffman W, Huckans MS (2011). Methamphetamine causes persistent immune dysregulation: a cross-species, translational report. Neurotox Res 20: 59-68.

London ED, Simon SL, Berman SM, Mandelkern MA, Lichtman AM, Bramen J et al (2004). Mood disturbances and regional cerebral metabolic abnormalities in recently abstinent methamphetamine abusers. Arch Gen Psychiatry 61: 73-84.
Mackillop J, Amlung MT, Few LR, Ray LA, Sweet LH, Munafo MR (2011). Delayed reward discounting and addictive behavior: a meta-analysis. Psychopharmacology (Berl) 216: 305-321.

Miller AH, Haroon E, Raison CL, Felger JC (2013). Cytokine targets in the brain: impact on neurotransmitters and neurocircuits. Depress Anxiety 30: 297-306.

Monterosso JR, Ainslie G, Xu J, Cordova X, Domier CP, London ED (2007). Frontoparietal cortical activity of methamphetaminedependent and comparison subjects performing a delay discounting task. Hum Brain Mapp 28: 383-393.

Narita M, Miyatake M, Narita M, Shibasaki M, Shindo K, Nakamura A et al (2006). Direct evidence of astrocytic modulation in the development of rewarding effects induced by drugs of abuse. Neuropsychopharmacology 31: 2476-2488.

Narita M, Miyatake M, Shibasaki M, Tsuda M, Koizumi S, Narita M et al (2005). Long-lasting change in brain dynamics induced by methamphetamine: enhancement of protein kinase C-dependent astrocytic response and behavioral sensitization. J Neurochem 93: 1383-1392.

Nunes EJ, Randall PA, Estrada A, Epling B, Hart EE, Lee CA et al (2014). Effort-related motivational effects of the pro-inflammatory cytokine interleukin 1-beta: studies with the concurrent fixed ratio 5/chow feeding choice task. Psychopharmacology (Berl) 231: 727-736.

Pometlová M, Nohejlová-Deykun K, Šlamberová R (2012). Anxiogenic effect of low-dose methamphetamine in the test of elevated plus-maze. Prague Med Rep 113: 223-230.

Reutskaja E, Hogarth RM (2009). Satisfaction in choice as a function of the number of alternatives: when "goods satiate". Psychol Mark 26: 197-203.

Roesch MR, Calu DJ, Schoenbaum G (2007). Dopamine neurons encode the better option in rats deciding between differently delayed or sized rewards. Nat Neurosci 10: 1615-1624.

Sekine Y, Ouchi Y, Sugihara G, Takei N, Yoshikawa E, Nakamura K et al (2008). Methamphetamine causes microglial activation in the brains of human abusers. J Neurosci 28: 5756-5761.

Shafiei N, Gray M, Viau V, Floresco SB (2012). Acute stress induces selective alterations in cost/benefit decision-making. Neuropsychopharmacology 37: 2194-2209.

Song C, Horrobin DF, Leonard BE (2006). The comparison of changes in behavior, neurochemistry, endocrine, and immune functions after different routes, doses and durations of administrations of IL-1beta in rats. Pharmacopsychiatry 39: 88-99.

Stellwagen D, Beattie EC, Seo JY, Malenka RC (2005). Differential regulation of AMPA receptor and GABA receptor trafficking by tumor necrosis factor-alpha. J Neurosci 25: 3219-3228.

Stolyarova A, O’Dell SJ, Marshall JF, Izquierdo A (2014). Positive and negative feedback learning and associated dopamine and serotonin transporter binding after methamphetamine. Behav Brain Res 271: 195-202.

Thijs LG, Hack CE (1995). Time course of cytokine levels in sepsis. Intensive Care Med 21(Suppl 2): S258-S263.

Turrin NP, Gayle D, Ilyin SE, Flynn MC, Langhans W, Schwartz GJ et al (2001). Pro-inflammatory and anti-inflammatory cytokine mRNA induction in the periphery and brain following intraperitoneal administration of bacterial lipopolysaccharide. Brain Res Bull 54: 443-453.

Vichaya EG, Hunt SC, Dantzer R (2014). Lipopolysaccharide reduces incentive motivation while boosting preference for high reward in mice. Neuropsychopharmacology 39: 2884-2890. 